\title{
Calvin Thomas Djombe, La fabrique des mauvaises filles. La cité et la construction de la " féminité déviante"
}

\author{
Jeanne Demoulin
}

1 À la fois livre de recherche et guide d'action à l'attention des travailleurs sociaux, $L a$ fabrique des mauvaises filles propose une réflexion sur l'étiquetage des jeunes filles des cités et ses conséquences sur leur intégration sociale. En s'appuyant sur son expérience d'éducateur dans les cités et sur la lecture d'écrits pluridisciplinaires, Calvin Thomas Djombe souhaite participer aux débats sur l'éducation des jeunes filles et démontrer que la cité et les codes de vie qui y règnent, en tant qu'ils sont dominés par le masculin, ont des conséquences négatives directes sur leur développement et leur comportement. Leur «féminité » devient en effet la cause de stigmatisations et de contraintes multiples au sein de leur famille et de la « cité ».

Pour ce faire, l'auteur décrit et analyse le processus d'étiquetage dont sont victimes des jeunes filles dans leurs familles, au sein de leur environnement proche et plus largement au sein de la société. Les termes de l'étiquetage mis en évidence par l'auteur sont tous liés au sexe et à la sexualité de ces dernières: "mauvaise fille", "fille souillée ", "pute ", «filles de banlieue » (p. 23) ou au contraire "femme pure », « oie blanche ", "corps pur », "vierge de toute souillure» (p. 55). Dans l'intimité comme dans l'espace public où les grands frères prennent le relais des parents (p.65), l'étiquetage vient sanctionner un comportement déviant ou, au contraire, ratifier un comportement adapté. Il participerait ainsi à contrôler les comportements des jeunes filles qui ajusteraient leurs manières pour éviter la stigmatisation.

D'après l'auteur, qui adopte alors une posture culturaliste, le fonctionnement des «familles immigrées» (p. 31 et suivantes), les modèles culturels des parents (en particulier portés par des pères présentés comme dominants et garants de l'honneur de la famille) et la manière dont ces familles transmettent les principes de la " distinction sexuelle» aux enfants (p. 43 et suivantes) permettent d'expliquer les attendus et les effets d'un tel processus d'étiquetage. Les familles exerceraient ainsi sur les jeunes filles 
une violence symbolique destinée à les « inciter à rester enfermées dans le carcan des règles de vie familiale» (p. 51). Celles qui cherchent à transgresser les règles ou à se différencier connaîtraient alors la « privation de liberté », la « culpabilité » et la « perte de dignité » (p. 51). Selon l'auteur, ce processus d'étiquetage conduit au développement d'une «sous-culture handicapante » (p.52) pour l'intégration sociale des jeunes filles, d'une «sujétion intériorisée » (p.154) qui les amènent à se disqualifier elles-mêmes pour certaines actions (p. 52).

4 C'est alors contre cet étiquetage que s'organise largement le travail des éducateurs : "Éduquer reviendra alors à " minimiser " l'étiquetage familial et environnemental » (p. 157). Au fil de l'ouvrage, l'auteur expose ainsi en contrepoint le chemin libérateur pour ces jeunes filles : la conquête de l'espace public et le fait de "sortir » de la cité, d'entrer dans la "société réelle » (p. 101). Il analyse cette " ambivalence » qui anime les jeunes filles, partagées entre l'envie de sortir de la cité, de s'extraire de l'autorité familiale tout en restant fortement attachés à la « culture d'origine » dans laquelle elles ont été éduquées (p.96). D’après l'auteur, cette sortie du «bunker» (p.121) que constituent la famille et la cité devient possible lorsqu'elles prennent conscience qu'elles ne pourront se construire que dans un contexte social plus élargi que celui de la cité (p. 61). Mais en sortant de la cité, projetées dans un environnement social et culturel qui leur est inconnu, les jeunes filles courent le risque de devoir tout réapprendre (p.125) et d'éprouver solitude, impuissance, vide relationnel. Les jeunes filles qui connaissent ce parcours chercheraient alors un "refuge »: " une nouvelle communauté », « une bande» (p.126), ou encore une «identité à la Janus » (p.141), adaptant leurs comportements en fonction de leur position géographique : « rangées au domicile, elles se "modernisent » et deviennent "sexy " dès qu'elles sortent de ce milieu et loin du regard des parents, des grands-frères et des autres jeunes de la cité ».

5 Si l'ouvrage propose une réflexion conceptuelle non dépourvue d'intérêt et nourrie par de nombreuses lectures, le flou autour de la méthodologie adoptée pour permettre la démonstration et la manière dont sont mobilisées les catégories centrales de l'ouvrage (les "cités", les "jeunes filles », leurs familles et leurs parents) limitent d'emblée l'interprétation des résultats avancés et la comparaison avec d'autres terrains d'enquêtes. Ainsi, les expériences de l'auteur comme éducateur ont certainement été riches et nombreuses mais celui-ci reste trop vague sur le corpus sur lequel s'appuie son enquête, que ce soit le profil des enquêtés ou les caractéristiques des "cités " étudiées. Il indique ainsi simplement: "notre aire d'enquête s'est focalisée essentiellement sur l'organisation de la vie dans certaines cités de la banlieue parisienne » (p. 15), dont certaines communes sont citées en note au début du premier chapitre (p. 22). Il ajoute un peu plus loin que «ce travail a exigé des enquêtes de terrain, une analyse des contextes sociaux, l'observation de la réalité humaine, familiale et sociale » ainsi que «l'exercice d'une réflexivité sur les actions personnelles de certains acteurs sociaux» (p.16). Deux des catégories centrales de l'ouvrage, les "cités » et les "jeunes filles", restent sans définition et il ressort de l'ouvrage une impression étrange : toutes les « cités » d'une part et toutes les « jeunes filles des cités » mais également leurs familles (et notamment les "parents immigrés») et leur environnement proche d'autre part semblent fonctionner exactement de la même manière.

6 Les jeunes filles ne sont dans le même temps considérées que de manière négative : à l'issue de la lecture, on ploie sous le fardeau qui pèse sur ces jeunes filles et leurs 
parents, les «problèmes » et « handicaps » multiples auxquels ils ont à faire face. Leurs quotidiens dans les "cités", "spirale infernale de descente aux enfers" et "vie quotidienne qui n'est qu'une mise au ban» (p. 61), ne sont pas plus attrayants. Mais le processus est-il si uniforme ? Sans négliger les difficultés à l'œuvre, n'existe-t-il pas des trajectoires moins accablantes? À faire du côté sombre une réalité unanime et partagée, le risque est de jouer en faveur de la stigmatisation de ces jeunes filles, et plus largement des territoires qu'elles habitent. Cela s'avèrerait selon nous plutôt contreproductif pour atteindre les objectifs éducatifs de nature émancipatrice annoncés par l'auteur.

$7 \quad$ La posture culturaliste adoptée et la manière de présenter les jeunes filles et les cités de manière très généralisatrice et homogénisante étonne d'autant plus que l'auteur connait la diversité des situations observables : « les trajectoires des jeunes filles de cité que rencontrent les éducateurs et travailleurs sociaux sont diverses, disparates, au point de s'opposer parfois, de même que leurs problématiques familiales » (p. 157) écrit-il dans le post-scriptum. On ne peut donc que rappeler pour conclure que la diversité des « cités » et des « jeunes filles » qui les habitent n'est plus à démontrer et qu'il apparait nécessaire de travailler avec cette complexité pour quiconque veut les penser ou intervenir auprès d'elles.

\section{AUTHOR}

\section{JEANNE DEMOULIN}

Sociologue et urbaniste, docteure en études urbaines, Post-doctorante au LAVUE, UMR CNRS

7218, au sein de l'ANR Pop-Part, Les quartiers populaires au prisme de la jeunesse. 